\title{
The Turbulent Network Dynamics of Microbial Evolution and the Statistical Tree of Life
}

\author{
Eugene V. Koonin ${ }^{1}$
}

Received: 18 March 2015/Accepted: 8 April 2015/Published online: 18 April 2015

(c) The Author(s) 2015. This article is published with open access at Springerlink.com

\begin{abstract}
The wide spread and high rate of gene exchange and loss in the prokaryotic world translate into "network genomics". The rates of gene gain and loss are comparable with the rate of point mutations but are substantially greater than the duplication rate. Thus, evolution of prokaryotes is primarily shaped by gene gain and loss. These processes are essential to prevent mutational meltdown of microbial populations by stopping Muller's ratchet and appear to trigger emergence of major novel clades by opening up new ecological niches. At least some bacteria and archaea seem to have evolved dedicated devices for gene transfer. Despite the dominance of gene gain and loss, evolution of genes is intrinsically tree-like. The significant coherence between the topologies of numerous gene trees, particularly those for (nearly) universal genes, is compatible with the concept of a statistical tree of life, which forms the framework for reconstruction of the evolutionary processes in the prokaryotic world.
\end{abstract}

Keywords Microbial evolution - Phylogenetic trees . Horizontal gene transfer · Muller's ratchet - Evolvability

\section{Introduction}

When in the late 1970s and early 1980s, Carl Woese and his colleagues constructed phylogenetic trees from 16S RNA sequence alignments, the resulting phylogenetic trees were

Eugene V. Koonin

koonin@ncbi.nlm.nih.gov

1 National Center for Biotechnology Information, National Library of Medicine, National Institutes of Health, Bethesda, MD 20894, USA thought to have solved the problem of microbial evolution (Woese 1987; Woese and Fox 1977; Woese et al. 1990). Indeed, all kinds of bacteria and the newly discovered domain of archaea were neatly classified in these trees, not withstanding some poorly resolved deep branches. However, this new order did not last long. As soon as the first few complete bacterial and archaeal genomes became available, comparative analysis of these sequences made it obvious that the 16S RNA tree told but a small part of the microbial evolution story (Doolittle 1999a, b). The evidence of the much greater complexity and a distinct character of microbial evolution has come from two complementary lines of observations: (i) the sequenced bacterial and archaeal genomes had dramatically different gene compositions, with only a small set of core genes being universally conserved (Koonin 2003; Perna et al. 2001); (ii) topologies of the numerous phylogenetic trees that became available for scrutiny with the advent of complete genomes were rarely fully compatible with the $16 \mathrm{~S}$ tree, and many of these trees were highly reliable indicating that the discrepancies could not be explained away by methodological artifacts alone (Koonin et al. 2001). Over the two decades that have passed since the sequencing of the first complete bacterial genomes, findings along these lines have led to a complete reappraisal of the nature of microbial evolution. The emerging understanding is that of an incessant flux of genes through genomes, or more precisely, pangenomes of microbes. The ability to accommodate new genes and even to donate genes to other microbes is likely to be an adaptive, evolvable function. Yet, all this does not necessarily imply that the tree of life has become an obsolete concept. In this brief review, I try to integrate different aspects of the "network genomics" of microbes in an attempt to outline, even if only in wide strokes, a new coherent concept of the microbial world evolution. 


\section{The Tree of Life is Dead: Long Live Phylogenetic Trees!}

The recent paradigm shift in the study of (microbial) genome evolution is most often discussed in terms of horizontal (lateral) gene transfer (HGT). Yet, the very concept of HGT is conditioned on the existence of a vertical, tree-like evolutionary standard (often referred to as the "tree of life") (Bapteste et al. 2005, 2009; Doolittle and Bapteste 2007). Explicitly or more often implicitly, the rRNA tree or a tree made from a concatenated alignment of several dozen (nearly) universal genes coding for components of the translation system is taken to represent such a standard (Ciccarelli et al. 2006; Woese 1987). However, these phylogenies have been quickly dubbed "trees of $1 \%$ " that reflected, at the very best, the evolution of a miniscule fraction of genes in each organism. As Dagan and Martin point out, a model that explains $1 \%$ of the data might be in need of replacement (Dagan and Martin 2006). Taking an even more radical view, Doolittle and colleagues have suggested that "tree thinking" in biology could be irrelevant to begin with, in particular because a tree easily can be used to depict similarity relationships between objects that have nothing to do with evolutionary relationships (Bapteste et al. 2005; Doolittle and Bapteste 2007).

Thus, the findings of microbial genomics have put into focus arguably the most basic question on evolution: is the tree of life simile touted by Darwin as the accurate depiction of the evolutionary process (Darwin 1859) a sheer illusion, at least as far as microbial evolution is concerned? I submit that this is not the case, and the "tree of life" remains a cornerstone of evolutionary biology although it has to be re-conceptualized in the light of the findings of evolutionary genomics. The argument is twofold, coming first from purely theoretical considerations and second, perhaps most important, from phylogenomic analysis. Conceptually, the history of cells is obviously a history of cell divisions and hence a tree-like process. More than that, genome replication is an inherently tree-like process as well; its tree structure is only disrupted by various forms of recombination that, however, can be quite frequent (as we discuss below). At sufficiently large evolutionary distances to eliminate homologous recombination, recombination within genes becomes deleterious and thus is rarely fixed, orders of magnitude less frequent than recombination between genes. Accordingly, gene evolution is an intrinsically tree-like process (Koonin and Wolf 2009).

With respect to genome evolution, the validity of the tree simile (using Darwin's language) remains an open question. The answer hinges on the existence of pronounced, coherent trends in the "phylogenetic forest," i.e., the entirety of individual gene trees. More specifically, does a tree of a universal gene reflects solely the evolutionary history of that gene or does it carry information on the evolution of other genes, and if so, how many genes and how much information? In a phylogenomic study that was specifically designed to address this question, my colleagues and I performed an exhaustive comparison of the topologies of thousands of phylogenetic trees of conserved eukaryotic genes (Puigbo et al. 2009, 2014). The results clearly indicate that the trees of the (nearly) universal genes, which encode primarily the translation system components, are not only highly consistent among themselves, but also with trees of numerous other genes. In quantitative terms, the consensus topology of the nearly universal trees (the notorious tree of $1 \%$ ) accounts for almost $40 \%$ of the variance in the tree topologies across the "forest" (Puigbo et al. 2010). Furthermore, this treelike signal reflecting the vertical inheritance of genetic information is by far the strongest trend in the "forest of life" because the remaining variance in tree topologies reflects largely the random gene exchange. Thus, the "tree of $1 \%$ " is not a failed hypothesis on genome evolution (Doolittle 2009) but rather a meaningful representation of the central current of genome evolution that can be legitimately construed as a "statistical tree of life" (STOL) (O'Malley and Koonin 2011) (Fig. 1). The STOL does not represent most (over $60 \%$ ) of the information flux that occurs during microbial evolution but it is the natural framework for reconstruction of these horizontal evolutionary currents.

\section{The Turbulent Dynamics of Microbial Evolution}

A key observation of microbial genomics is that the genomes of organisms that are very closely related in terms of the sequence similarity of the universal genes (e.g., have

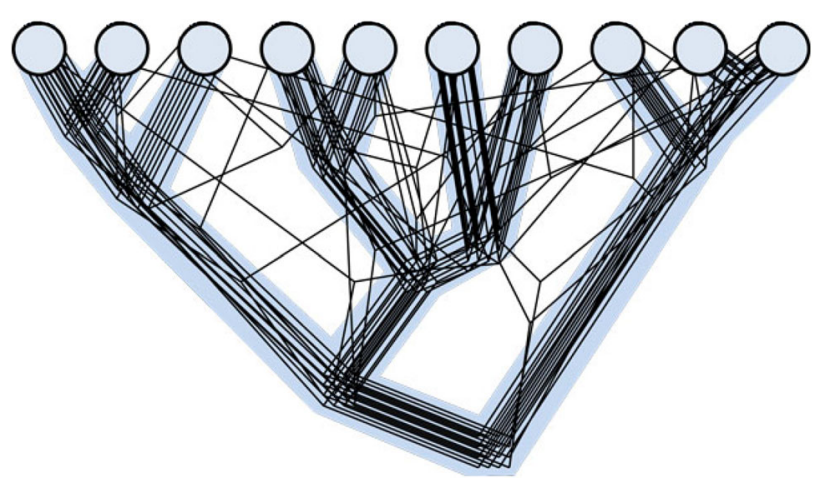

Fig. 1 The statistical tree of life. The gray background shows the central vertical trend. The depicted "forest of life" consists of 16 trees with 20 deviations from the central trend. Reproduced from (Puigbo et al. 2013) the Creative Commons Attribution License 
identical 16S RNA sequences) often substantially differ in their gene repertoires (Perna et al. 2001). Thus, comparative genome analysis can be informative of both the patterns and the dynamics of genome evolution. The observations on the strong vertical evolution trend in the "forest of life" described above provide justification for the use of the tree of universal genes as a scaffold for evolutionary reconstruction (usually known as species tree). For groups of microbes at the species or genus level, such trees can be highly accurate. Given a species tree, all the genes in the pangenome of a species or an otherwise defined group of microbes (i.e., the entirety of the genes represented in the available isolates of the given group) can be mapped to the leaves of the tree. Thus, mapping then can be used to reconstruct the evolutionary scenario for the pangenome, i.e., the history of gene gains, losses, and duplications. In the early days of evolutionary genomics, such reconstructions were performed using the simple parsimony approaches that select the scenario with the minimum number of events (Kunin and Ouzounis 2003; Mirkin et al. 2003; Snel et al. 2002). Subsequently, more sophisticated maximum likelihood methods have been developed that employ evolutionary birth-and-death models to derive statistical estimates for the number of different genomic events associated with each branch of the species tree (Csuros 2010; Csuros and Miklos 2009).

A recent application of the maximum likelihood approach to the reconstruction of evolution for diverse groups of closely related bacteria (and one archaeal group) has revealed a striking picture of genomes in turmoil (Puigbo et al. 2014). Although the rates of gene gain, loss, and duplication differ by orders of magnitude across the bacterial diversity, in the most dynamic groups, several gains and losses can occur during the time that takes for the genome to accumulate, on average, one nucleotide substitution per gene. A further unexpected finding is that the most common process of genome dynamics is actually loss of genes. The estimates indicate that there are two to three times more losses than gains per nucleotide substitution (used in this case as the unit of time). Clearly, in the long term, excess of gene losses would lead to genome degradation and eventually extinction, and such is indeed the fate of many lineages, in particular those including parasites and symbionts (Merhej et al. 2013). More generally, however, the gradual gene loss seems to be off-set by bursts of gene gain that might accompany the emergence of major, phyla level and higher, groups of prokaryotes (see more below on such bursts of innovation) (Wolf and Koonin 2013). Remarkably, the rates of gene loss and gain are at least an order of magnitude greater than the gene duplication rate (Puigbo et al. 2014; Treangen and Rocha 2011).

The observations on the dynamics of microbial genome evolution clearly show that, at least in this part of the biosphere, evolution does not primarily proceed via the Darwinian route codified in the Modern Synthesis of Evolutionary Biology, i.e., by accumulation of numerous, "infinitesimally small" beneficial changes (mutations) (Darwin 1859; Dobzhansky 1937) but rather by much bigger, at least gene-sized, leaps. Furthermore, in bacteria and archaea, the dominant of genome dynamics is not "evolution by gene duplication" (Lynch and Conery 2000; Ohno 1970) that appears to be so prominent in eukaryotes, but rather, evolution by gene gain and loss.

\section{Pangenomes and Supergenomes of Microbes: Are There Limits to Innovation?}

The discoveries of the frequent major differences between closely related microbes and the extensive gene gain that shapes the genomes of archaea and bacteria have changed the paradigm of microbial genomics. We now realize that the genome isolated from a bacterial colony is not a stable "blueprint" of the organism but rather a transient gene collection that, on the evolutionary timescale, can rapidly gain or lose a substantial fraction of those genes. Thus, the more relevant concepts in microbial evolutionary genomics are pangenome and supergenome (Land et al. 2015; Tettelin et al. 2005, 2008). It makes sense to differentiate between the two (Puigbo et al. 2014). The pangenome is the entirety of the genes discovered in the sequenced genomes of all isolates of a given microbial species (how to define a microbial species and even whether the notion of species makes sense for microbes, is unclear (Doolittle and Zhaxybayeva 2009); nevertheless, thousands of bacterial and archaeal genes are formally recognized, and for the sake of simplicity, I discuss pangenomes and supergenomes at the species level although in principle, both can be defined for any group of organisms). The pangenome thus is a moving target, and its size can increase with each sequenced isolate (Bosi et al. 2015). For the majority of the extensively sequenced bacterial and archaeal genomes, this is indeed the case, and signs of saturation of the number of gene are not (yet) apparent (Fig. 2). Such growing pangenomes are often called "open." Some microbes, however, have closed pangenomes that saturate after only a few isolates are sequenced; a notable case of a closed pangenome is the (in) famous pathogen Bacillus anthracis (Tettelin et al. 2008).

The supergenome can be defined as the entirety of the genes that are accessible for gain to the isolates of a given species. In principle, the supergenome and the pangenome become one and the same when all isolates on earth are sequenced, i.e., the supergenome is the limit to which the pangenome tends (Fig. 2). In practice, obviously, the supergenome cannot be characterized directly, and its size 
Fig. 2 Microbial pangenomes and supergenomes. The figure schematically shows the growth of the pangenome for three types of supergenomes: small, closed (pangenome saturates after only a few genomes are sequenced); larger, closed (pangenome approaches saturation, i.e., the supergenome, as the number of genomes increases from 1 to 20 ); and open (no sign of saturation)

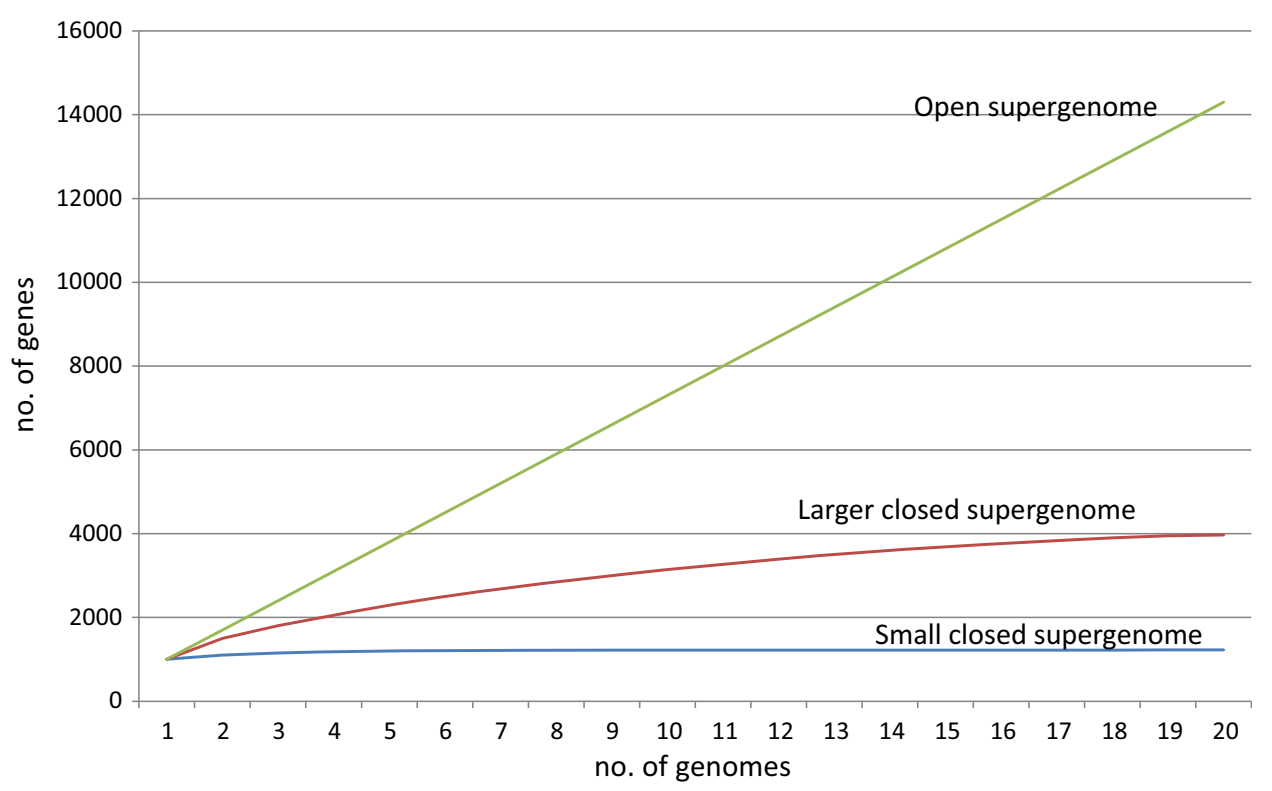

has to be inferred from the available genomic data (Baumdicker et al. 2012; Bosi et al. 2015; Collins and Higgs 2012; Lobkovsky et al. 2014). Several mathematical models have been developed for this purpose. Conceivably, the simplest approach infers the supergenome size from the number of repeated gains of the same gene family detected in isolates of the same species. Intuitively, if the same genes are gained all the time, the supergenome is quite small, whereas if all gains are unique, the supergenome is operationally infinite. A maximum likelihood estimation of the supergenome size for a variety of bacteria based on this simple approach has yielded surprisingly consistent estimates of supergenomes exceeding the typical size of the genome for the given species about tenfold. In some groups, however, the supergenomes did appear "infinite" (Puigbo et al. 2014). Given the current limited sampling of the microbial world and our still crude understanding of the patterns of gene flux, these supergenome size estimates certainly should be viewed as preliminary (Lobkovsky et al. 2014). However, the estimates as low as ten genomic equivalents will soon be put to test for many groups of bacteria and archaea.

The gene exchange within microbial supergenomes translates into a characteristic distribution of gene frequencies in pangenomes that is remarkably well reproduced across a wide range of phylogenetic depths, from individual species to large sets of organisms representing the entire known diversity of archaea and bacteria (Koonin and Wolf 2008; Lobkovsky et al. 2013). This distribution includes three distinct components of vastly different sizes: (i) the conserved core of (nearly) universal genes that represents a small minority of the pangenomes (it is these genes, coding primarily for components of information processing systems, that give rise to the "tree of $1 \%$ "); (ii) the moderately conserved "shell" that consists, to a large extent, of genes encoding metabolic enzymes and transport systems; and (iii) the "cloud" of rare genes that encode signaling molecules, defense systems, and a huge number of uncharacterized proteins (Fig. 3). The size of the rare gene cloud, like the size of the supergenome, is unknown but obviously, vastly exceeds the size of the shell. This tripartite distribution is an invariant in the genome universe and is, to a large extent, shaped by selectively neutral processes of gene flux. However, mathematical modeling of genome evolution shows that strictly neutral genome evolution would not produce the observed fraction of the highly conserved gene that constitute the core and much of the shell (Lobkovsky et al. 2013). The extent of the

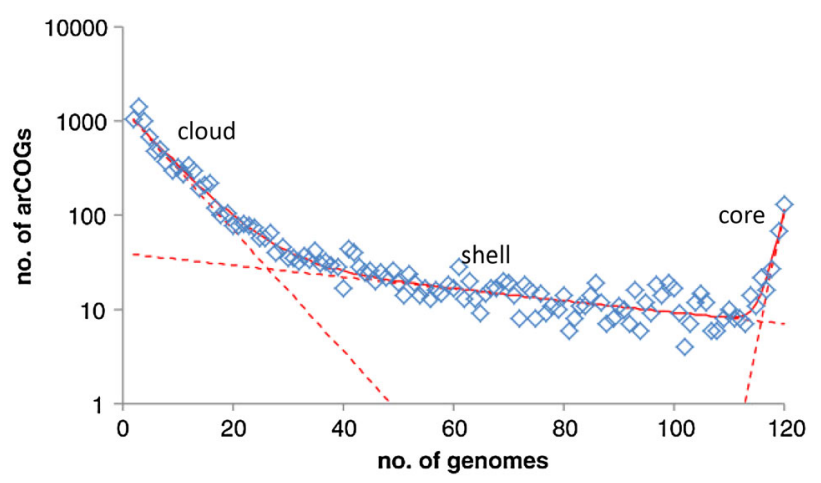

Fig. 3 The universal distribution of gene frequencies. The plot shows gene frequencies for 120 archaeal genomes. The dashed lines show the three exponents that approximate the core, the shell, and the cloud. The solid line shows the sum of the three functions. Modified from (Wolf et al. 2012) the Creative Commons Attribution License 
evolutionary conservation of these genes implies selection stemming from their unique functional capacities.

\section{The Evolutionary Impact and Adaptive Value of Horizontal Gene Transfer: Is Horizontal Gene Transfer Evolvable?}

Could microbes evolve without horizontal gene transfer, simply via the competition of stable, clonal populations? Population genetic analysis indicates that such evolutionary regime is unsustainable in the long term (Takeuchi et al. 2014). Finite size clonal population typically deteriorate due to the action of the evolutionary mechanism known as Muller's ratchet that involves accumulation of slightly deleterious mutations due to genetic drift resulting in gradual loss of fitness and eventual extinction (Charlesworth et al. 1993; Muller 1964). This appears to be the typical fate of bacteria that are confined to intracellular parasitism or symbiosis, although the action of the ratchet could be slowed down by lowering mutation rate (Allen et al. 2009). However, such mechanisms hardly can stop the ratchet altogether. It appears that the only path of escape from the Muller's ratchet doom is gene acquisition via HGT that can result either in displacement of a mutated gene by a functional copy or by acquisition of new genes that offsets the deleterious effects of accumulating mutations (Takeuchi et al. 2014). Clearly, in prokaryotes, HGT plays the same role of preventing mutational meltdown that in eukaryotes is played by sex (Ku et al. 2015).

Escape from Muller's ratchet could be, in a sense, the most basic role of HGT in microbial evolution but it certainly is not the only one. Acquisition of new genes and whole suits of genes, such as operons, appears to be the principal way of expanding metabolic networks in microbes (Andersson 2009; Treangen and Rocha 2011). Furthermore, as the network grows, gain of only one enzyme is increasingly likely to be beneficial, by providing access to a new nutrient (Maslov et al. 2009).

Massive gene gain via HGT appears to be the driving force behind the origin of major groups of organisms. Recent extensive search of archaeal genomes for acquired bacterial genes suggests that the emergence of most if not all major archaeal clades was associated with and conceivably caused by acquisition of hundreds or even thousands of bacterial genes (Nelson-Sathi et al. 2015). The largest influx of bacterial genes was detected in mesophilic groups such as Halobacteria and Methanobacteria and apparently led to fundamental innovation, i.e., adaptation to new lifestyles and ecological niches (Nelson-Sathi et al. 2012, 2015). The eukaryotes evolved via the same scenario, with the obvious, important distinction that the bacterial donor of the acquired genes was preserved in the form of the proto-mitochondrial endosymbiont $(\mathrm{Ku}$ et al. 2015).

Is HGT an evolvable capacity or in other words, an adaptive, selectable trait? Despite the wide spread and essential role of HGT in microbial evolution, this is not a trivial question because potentially HGT could be considered a neutral consequence of the presence of substantial amounts of DNA in the environment and of genetic processes such as bacteriophage infection that lead to gene transfer (transduction) (Bushman 2001). Numerous bacteria and archaea are competent for natural transformation that is mediated by specialized DNA intake pumps (Claverys et al. 2009). In principle, these pumps can be viewed as devices for utilization of environmental DNA as a source of nucleotides, with HGT being a fringe benefit. However, the recent demonstration that in some bacteria, the ingested DNA is specifically protected against degradation, thus facilitating HGT, implies that at least in part, natural competence evolved as a gene transfer machinery (Johnston et al. 2013). Bacterial conjugation (prokaryotic sex) appear to be another dedicated mechanism of gene transfer but this route involves only very closely related isolates and, similar to the eukaryotic sex, could be viewed as an evolutionary mechanism to escape from Muller's ratchet (Ku et al. 2015).

At present, perhaps, the best showcase for dedicated vehicles of HGT appears to be the gene transfer agents (GTAs). The GTAs are defective prophages that form virus particles in which, however, they package apparently random fragments of the bacterial chromosome, rather than the phage genome (Lang et al. 2012). The GTAs then infect other bacteria or archaea, and the transferred DNA integrates into the recipient genome. In marine bacterial communities, the rate of gene transfer appears to be quite high and often involves distantly related organisms (McDaniel et al. 2010). A remarkable aspect of the GTAs is that they confer onto their carriers the ability to donate rather than acquire genetic material. Such a capacity could be adaptive in the context of utilization of "public goods" by microbial communities. The wide spread of GTAs appears to present strong evidence of evolvability of HGT.

\section{Concluding Remarks}

The wide spread and high rate of gene exchange and loss in the prokaryotic world translate into "network genomics." These processes are essential to prevent mutational meltdown in microbial populations (stop Muller's ratchet) and are key contributors to innovation including origin of new clades with novel lifestyles. The contribution of gene gain and loss in microbial evolution is ostensibly greater than the contribution of point mutations. The strongest 
indication of the importance of massive gene transfer for the emergence of major clades comes from comparative genomics of archaea where influx of bacterial genes seems to have coincided with the origin of multiple phyla. The eukaryotes apparently evolved via a similar scenario, with the crucial distinction of the survival of the bacterial gene donor in the form of an endosymbiont. Bacteria and archaea appear to have evolved multiple dedicated devices for gene transfer.

Not withstanding the ubiquity and essentiality of gene transfer, tree-like processes are intrinsic to the processes of replication and cell division. Moreover, the substantial coherence between the topologies of numerous gene trees, particularly those for (nearly) universal genes, is compatible with the concept of a statistical tree of life, a central vertical trend in genome evolution. The statistical tree of life is a natural framework for the reconstruction of processes of gene gain and loss that shape the evolution of the prokaryotic world.

Acknowledgments The author's research is supported by intramural Funds of the US Department of Health and Human Services (to the National Library of Medicine).

Open Access This article is distributed under the terms of the Creative Commons Attribution 4.0 International License (http:// creativecommons.org/licenses/by/4.0/), which permits unrestricted use, distribution, and reproduction in any medium, provided you give appropriate credit to the original author(s) and the source, provide a link to the Creative Commons license, and indicate if changes were made.

\section{References}

Allen JM, Light JE, Perotti MA, Braig HR, Reed DL (2009) Mutational meltdown in primary endosymbionts: selection limits Muller's ratchet. PLoS One 4:e4969

Andersson JO (2009) Gene transfer and diversification of microbial eukaryotes. Annu Rev Microbiol 63:177-193

Bapteste E, Susko E, Leigh J, MacLeod D, Charlebois RL, Doolittle WF (2005) Do orthologous gene phylogenies really support treethinking? BMC Evol Biol 5:33

Bapteste E, O'Malley MA, Beiko RG, Ereshefsky M, Gogarten JP, Franklin-Hall L, Lapointe FJ, Dupre J, Dagan T, Boucher Y et al (2009) Prokaryotic evolution and the tree of life are two different things. Biol Direct 4:34

Baumdicker F, Hess WR, Pfaffelhuber P (2012) The infinitely many genes model for the distributed genome of bacteria. Genome Biol Evol 4:443-456

Bosi E, Fani R, Fondi M (2015) Defining orthologs and pangenome size metrics. Methods Mol Biol 1231:191-202

Bushman F (2001) Lateral DNA transfer: mechanisms and consequences. Cold Spring Harbor Laboratory Press, New York

Charlesworth D, Morgan MT, Charlesworth B (1993) Mutation accumulation in finite outbreeding and inbreeding populations. Genet Res 61:39-56

Ciccarelli FD, Doerks T, von Mering C, Creevey CJ, Snel B, Bork P (2006) Toward automatic reconstruction of a highly resolved tree of life. Science 311:1283-1287
Claverys JP, Martin B, Polard P (2009) The genetic transformation machinery: composition, localization, and mechanism. FEMS Microbiol Rev 33:643-656

Collins RE, Higgs PG (2012) Testing the infinitely many genes model for the evolution of the bacterial core genome and pangenome. Mol Biol Evol 29:3413-3425

Csuros M (2010) Count: evolutionary analysis of phylogenetic profiles with parsimony and likelihood. Bioinformatics 26: 1910-1912

Csuros M, Miklos I (2009) Streamlining and large ancestral genomes in Archaea inferred with a phylogenetic birth-and-death model. Mol Biol Evol 26:2087-2095

Dagan T, Martin W (2006) The tree of one percent. Genome Biol $7: 118$

Darwin C (1859) On the origin of species, 1st edn. Murray, London

Dobzhansky T (1937) Genetics and the origin of species. Columbia University Press, New York

Doolittle WF (1999a) Lateral genomics. Trends Cell Biol 9:M5-M8

Doolittle WF (1999b) Phylogenetic classification and the universal tree. Science 284:2124-2129

Doolittle WF (2009) The practice of classification and the theory of evolution, and what the demise of Charles Darwin's tree of life hypothesis means for both of them. Philos Trans R Soc Lond B Biol Sci 364:2221-2228

Doolittle WF, Bapteste E (2007) Pattern pluralism and the tree of life hypothesis. Proc Natl Acad Sci USA 104:2043-2049

Doolittle WF, Zhaxybayeva O (2009) On the origin of prokaryotic species. Genome Res 19:744-756

Johnston C, Polard P, Claverys JP (2013) The DpnI/DpnII pneumococcal system, defense against foreign attack without compromising genetic exchange. Mob Genet Elements 3:e25582

Koonin EV (2003) Comparative genomics, minimal gene-sets and the last universal common ancestor. Nat Rev Microbiol 1:127-136

Koonin EV, Wolf YI (2008) Genomics of bacteria and archaea: the emerging dynamic view of the prokaryotic world. Nucleic Acids Res 36:6688-6719

Koonin EV, Wolf YI (2009) The fundamental units, processes and patterns of evolution, and the tree of life conundrum. Biol Direct 4:33

Koonin EV, Makarova KS, Aravind L (2001) Horizontal gene transfer in prokaryotes: quantification and classification. Annu Rev Microbiol 55:709-742

Ku C, Nelson-Sathi S, Roettger M, Garg S, Hazkani-Covo E, Martin WF (2015) Endosymbiotic gene transfer from prokaryotic pangenomes: inherited chimerism in eukaryotes. Proc Natl Acad Sci USA. doi:10.1073/pnas.1421385112

Kunin V, Ouzounis CA (2003) The balance of driving forces during genome evolution in prokaryotes. Genome Res 13:1589-1594

Land M, Hauser L, Jun SR, Nookaew I, Leuze MR, Ahn TH, Karpinets T, Lund O, Kora G, Wassenaar T et al (2015) Insights from 20 years of bacterial genome sequencing. Funct Integr Genomics 15:141

Lang AS, Zhaxybayeva O, Beatty JT (2012) Gene transfer agents: phage-like elements of genetic exchange. Nat Rev Microbiol 10:472-482

Lobkovsky AE, Wolf YI, Koonin EV (2013) Gene frequency distributions reject a neutral model of genome evolution. Genome Biol Evol 5:233-242

Lobkovsky AE, Wolf YI, Koonin EV (2014) Estimation of prokaryotic supergenome size and composition from gene frequency distributions. BMC Genomics 15(Suppl 6):S14

Lynch M, Conery JS (2000) The evolutionary fate and consequences of duplicate genes. Science 290:1151-1155

Maslov S, Krishna S, Pang TY, Sneppen K (2009) Toolbox model of evolution of prokaryotic metabolic networks and their regulation. Proc Natl Acad Sci USA 106:9743-9748 
McDaniel LD, Young E, Delaney J, Ruhnau F, Ritchie KB, Paul JH (2010) High frequency of horizontal gene transfer in the oceans. Science 330:50

Merhej V, Georgiades K, Raoult D (2013) Postgenomic analysis of bacterial pathogens repertoire reveals genome reduction rather than virulence factors. Brief Funct Genomics 12:291-304

Mirkin BG, Fenner TI, Galperin MY, Koonin EV (2003) Algorithms for computing parsimonious evolutionary scenarios for genome evolution, the last universal common ancestor and dominance of horizontal gene transfer in the evolution of prokaryotes. BMC Evol Biol 3:2

Muller HJ (1964) The relation of recombination to mutational advance. Mutat Res 106:2-9

Nelson-Sathi S, Dagan T, Landan G, Janssen A, Steel M, McInerney JO, Deppenmeier U, Martin WF (2012) Acquisition of 1,000 eubacterial genes physiologically transformed a methanogen at the origin of Haloarchaea. Proc Natl Acad Sci USA 109:2053720542

Nelson-Sathi S, Sousa FL, Roettger M, Lozada-Chavez N, Thiergart T, Janssen A, Bryant D, Landan G, Schonheit P, Siebers B et al (2015) Origins of major archaeal clades correspond to gene acquisitions from bacteria. Nature 517:77-80

Ohno S (1970) Evolution by gene duplication. Springer, New York

O'Malley MA, Koonin EV (2011) How stands the tree of life a century and a half after the origin? Biol Direct 6:32

Perna NT, Plunkett G 3rd, Burland V, Mau B, Glasner JD, Rose DJ, Mayhew GF, Evans PS, Gregor J, Kirkpatrick HA et al (2001) Genome sequence of enterohaemorrhagic Escherichia coli O157:H7. Nature 409:529-533

Puigbo P, Wolf YI, Koonin EV (2009) Search for a tree of life in the thicket of the phylogenetic forest. J Biol 8:59

Puigbo P, Wolf YI, Koonin EV (2010) The tree and net components of prokaryote evolution. Genome Biol Evol 2:745-756

Puigbo P, Wolf YI, Koonin EV (2013) Seeing the tree of life behind the phylogenetic forest. BMC Biol 11:46
Puigbo P, Lobkovsky AE, Kristensen DM, Wolf YI, Koonin EV (2014) Genomes in turmoil: quantification of genome dynamics in prokaryote supergenomes. BMC Biol 12:66

Snel B, Bork P, Huynen MA (2002) Genomes in flux: the evolution of archaeal and proteobacterial gene content. Genome Res $12: 17-25$

Takeuchi N, Kaneko K, Koonin EV (2014) Horizontal gene transfer can rescue prokaryotes from Muller's ratchet: benefit of DNA from dead cells and population subdivision. G3 (Bethesda) $4: 325-339$

Tettelin H, Masignani V, Cieslewicz MJ, Donati C, Medini D, Ward NL, Angiuoli SV, Crabtree J, Jones AL, Durkin AS et al (2005) Genome analysis of multiple pathogenic isolates of Streptococcus agalactiae: implications for the microbial "pan-genome". Proc Natl Acad Sci USA 102:13950-13955

Tettelin H, Riley D, Cattuto C, Medini D (2008) Comparative genomics: the bacterial pan-genome. Curr Opin Microbiol 11: 472-477

Treangen TJ, Rocha EP (2011) Horizontal transfer, not duplication, drives the expansion of protein families in prokaryotes. PLoS Genet 7:e1001284

Woese CR (1987) Bacterial evolution. Microbiol Rev 51:221-271

Woese CR, Fox GE (1977) Phylogenetic structure of the prokaryotic domain: the primary kingdoms. Proc Natl Acad Sci USA 74: 5088-5090

Woese CR, Kandler O, Wheelis ML (1990) Towards a natural system of organisms: proposal for the domains archaea, bacteria, and eucarya. Proc Natl Acad Sci USA 87:4576-4579

Wolf YI, Koonin EV (2013) Genome reduction as the dominant mode of evolution. Bioessays 35:829-837

Wolf YI, Makarova KS, Yutin N, Koonin EV (2012) Updated clusters of orthologous genes for archaea: a complex ancestor of the archaea and the byways of horizontal gene transfer. Biol Direct $7: 46$ 